\title{
Effects of paired and unpaired trials beyond the peak CR on acquisition and extinction of a classically conditioned SCR
}

\author{
JOHN C. MOREY, ROBERT J. MCCAFFREY, and AVRUM I. SILVER \\ University of Georgia, Athens, Georgia 30602
}

\begin{abstract}
The present experiment investigated the postpeak CR attenuation process during classical conditioning of the SCR and its relationship to subsequent extinction. Either paired trials were continued or unpaired trials were introduced following a subject's peak CR for an additional 2 or 16 trials. Two control groups received uniformly unpaired presentations of the CS and the UCS. All six groups were then given extinction trials. Stronger resistance to extinction with 2 as contrasted to 16 acquisition trials was demonstrated only with the postpeak paired group. Disassociation of the CS and the UCS beyond the peak CR eliminated the effects of conditioning during extinction. Strong resistance to extinction, therefore, appears to be associated with very few acquisition trials past the peak CR, and maximum CR strength is achieved if those trials are paired rather than unpaired. An acceleration of the CR attenuation process during postpeak unpaired trials and traces of a strong UCS arousal response were offered as explanations for the results.
\end{abstract}

Since the skin conductance response (SCR) is susceptible to rapid habituation, large responses tend to be temporary and attenuation of the conditioned response (CR) during classical conditioning of the SCR appears as a rather consistent finding (Kimmel, 1966; Silver, 1977). Following a classical conditioning procedure, moreover, strong resistance to extinction rarely has been observed when the SCR has been used as the response measure. However, a number of recent studies have shown relatively strong persistence of the $\mathrm{CR}$, which now necessitates a closer examination of the $\mathrm{CR}$ attenuation process. $^{1}$

In one of these studies by Silver and Kimmel (1969), a novel technique was employed in which strong resistance to extinction of the CR was demonstrated. Since the CR in classical SCR conditioning reaches a peak magnitude early and then becomes attenuated, Silver and Kimmel varied the number of paired presentations beyond the peak. Subjects receiving 2 paired trials beyond the peak continued to respond for a total of 42 trials (i.e., the number of paired trials plus extinction trials beyond the peak); subjects who received 16 postpeak paired trials responded for only 30 trials. An even greater difference occurred in a subsequent study (Schramm \& Kimmel, 1970), in that the 2-postpeak group responded for 46 trials, compared with 24 trials for the 16-postpeak group. Generally, both studies demonstrated an inverse relationship between the number of paired trials beyond the peak and resistance to extinction.

Requests for reprints should be addressed to John C. Morey, Army Research Institute Field Unit, P.O. Box 2086, Fort Benning, Georgia 31905.
During extinction, the CR typically becomes attenuated and may reach a zero response amplitude. While response attenuation is also characteristic of postpeak acquisition during conditioning, it might be expected to occur more rapidly during extinction, since the unconditioned stimulus (UCS) is no longer presented. If extinction were introduced just after the peak CR, the expectation is that more rapid attenuation should result than with continuation of the conditioned stimulus (CS)/UCS pairing. However, no evidence exists for this, but there is reason to believe that postpeak extinction trials result in greater persistence of the CR than do paired trials.

At the very least, extinction trials just beyond the peak might reveal no more $C R$ attenuation than do paired trials. One explanation involves the possibility that trials beyond the peak, be they CS alone (i.e., extinction), paired, or even unpaired, simply produce the same degree of response decrement. The implication is that the habituation process is the same no matter what procedure is used. To test this implication, postpeak unpaired presentations of the CS and UCS are included in the present study as a separate condition, together with a condition employing the previously used postpeak paired trials. Extinction introduced just past the peak provides an additional condition.

In the Schramm and Kimmel (1970) and Silver and Kimmel (1969) studies, giving just 2 paired trials beyond the peak produced significantly more resistance to extinction than giving 16 trials beyond the peak. To what extent this will occur with unpaired trials beyond the peak is examined in the present study, along with an analysis of postpeak CR magnitudes. If response strength (i.e., CR magnitude) is .found to be as strong during 
extinction just following the peak as it is during pairing just beyond the peak, then this strength during extinction could be related to one of two characteristics inherent in the extinction procedure: the nonassociation of the UCS and the CS and the removal of the UCS. A comparison of the postpeak paired and unpaired CR magnitudes was undertaken to elucidate the relevant characteristics of the postpeak process.

\section{METHOD}

\section{Subjects}

The subjects were 84 undergraduates who participated as part of a course requirement at the University of Georgia. Fourteen subjects were randomly assigned to each of six conditions.

\section{Apparatus}

The CS was a $39-\mathrm{dB} 1,000 \mathrm{~Hz}$ tone of $5.0 \mathrm{sec}$ duration delivered from an RCA audio generator to a loudspeaker placed $1 \mathrm{~m}$ to the right of the subject. The electric shock UCS was produced by an Argonaut Associates constant-current amplifier (Model LRA046) and delivered to the volar surface of the subject's right forearm via copper electrodes. Intensity of the UCS was $4.0 \mathrm{~mA}$ with a duration of $.1 \mathrm{sec}$, occurring $5.0 \mathrm{sec}$ after CS onset. Skin resistance was recorded from the palm and back of the subject's left hand by $2.84-\mathrm{cm}^{2}$ zinc/zinc sulfate electrodes in lucite cups filled with a zinc sulfate electrolyte in an agar-agar paste. Current density was constant at $10 \mathrm{~mA} / \mathrm{cm}^{2}$. Tonic (SRL) and phasic (SRR) skin resistance changes were amplified on separate channels by an Electronics Laboratories amplifier (Model 308C) and recorded on a dual-channel Texas Instruments Recti-Riter with a paper speed of $.127 \mathrm{~cm} / \mathrm{sec}$. Subjects were seated in a standard office chair in a darkened, sound-attenuating Industrial Acoustics Company chamber. The experimenter communicated with the subject through a two-way intercom.

\section{Procedure}

All subjects initially received three presentations of the UCS in increasing intensities of $1.0,2.5$, and $4.0 \mathrm{~mA}$ dc (i.e., sensitization), followed by 12 habituation trials of the CS (Silver, 1973). Fifty-six of the subjects then received paired presentations of the CS and UCS, each subject being presented paired trials until the maximal (peak) SCR had been obtained. The subject immediately continued with one of the four following procedures: (1) 2PP-P-CS and UCS paired for 2 additional trials beyond peak SCR, followed by CS-only trials; (2) 2PP-UnP-CS and UCS unpaired for 2 additional trials beyond peak SCR, followed by CS-only trials; (3) $16 \mathrm{PP}-\mathrm{CS}$ and UCS paired for 16 additional trials beyond peak SCR, followed by CS-only trials; (4) 16PP-UnP-CS and UCS unpaired for 16 additional trials beyond peak SCR, followed by CS-only trials.

The remaining 28 subjects served as sensitization controls (2PP-SC and 16PP-SC) yoked to the 2PP-P and 16PP-P conditions. Each control subject received the same number of $\mathrm{CS}$ and UCS presentations as the yoked partner, but with the restriction that the stimuli were not paired. The CS was then presented alone (extinction). Extinction criterion for all six conditions was two consecutive nonresponses or 50 trials, whichever came first. A response was designated a CR if it occurred within the interval of $1.5-6.5 \mathrm{sec}$ following CS onset. The intertrial interval ranged from 15 to $45 \mathrm{sec}$, with a mean of $30 \mathrm{sec}$. The rejection region adopted for all statistical comparisons was $p<.05$.

\section{RESULTS}

To determine if conditioning had occurred, comparisons were made early during acquisition because of the rapid attenuation of the CR beyond the peak. Only the first three trials of acquisition were used for this comparison, since unpaired trials for some subjects were introduced on the third trial, although use of the third trial was permissible since the effects of unpairing on the CR could not be observed until the fourth trial. Up through the presentation of the CS on the third trial, all subjects received either paired or unpaired presentations of the CS and UCS uncontaminated by the other conditions introduced later. Therefore, all subjects who received paired trials were compared with all subjects who received unpaired trials. This unequal-n comparison involved the magnitude of the response made to the CS for the first three acquisition trials. The subjects in the paired group made larger responses to the CS than the subjects in the unpaired group. The paired group also demonstrated increasingly larger CRs than did the unpaired group across the three acquisition trials. The main effects for group $[\mathrm{F}(1,82)=4.48, \mathrm{MSe}=.0008]$ and trials $[F(2,164)=6.33$, MSe $=.0003]$ were significant. The Group by Trials interaction also was significant $[F(2,164)=18.67, \mathrm{MSe}=.003]$.

The extinction data are presented in Table 1 and reveal mean trials to criterion and standard deviations for all groups. With previous studies, large differences in resistance to extinction were obtained between the 2- and 16-postpeak paired conditions, and the same effect was observed in the present study. These differences are somewhat reduced in the postpeak unpaired groups and nonexistent with the completely unpaired conditions. Pairing beyond the peak did result in a statistically significant difference between the 2 and 16 conditions $[\mathrm{t}(26)=2.12]$. Unpaired presentations beyond the peak failed to result in significant extinction differences between the two conditions, as was true of the completely unpaired groups.

The data in Table 1 also show differences when postpeak paired and unpaired conditions are compared with the completely unpaired control groups. This kind of comparison can be viewed as a measure of the effect of conditioning during extinction. Within the twopostpeak condition, the group that received paired trials beyond the peak showed the largest difference from the control. The difference between the control and the 2-postpeak unpaired condition was somewhat reduced, and little or no differences were observed within the "16" conditions. An overall analysis of variance revealed

Table 1

Mean Number of Trials to Criterion During Extinction for All Groups

\begin{tabular}{|c|c|c|c|c|c|c|}
\hline \multirow[b]{3}{*}{$\mathbf{T}$} & \multicolumn{6}{|c|}{ Paired/Unpaired Condition } \\
\hline & \multicolumn{2}{|c|}{$\begin{array}{l}\text { Postpeak } \\
\text { Paired }\end{array}$} & \multicolumn{2}{|c|}{$\begin{array}{l}\text { Postpeak } \\
\text { Unpaired }\end{array}$} & \multicolumn{2}{|c|}{$\begin{array}{c}\text { Completely } \\
\text { Unpaired }\end{array}$} \\
\hline & Mean & SD & Mean & SD & Mean & SD \\
\hline 2 & 29.14 & 18.02 & 22.29 & 14.32 & 11.21 & 15.69 \\
\hline 16 & 15.43 & 16.23 & 12.21 & 12.99 & 11.36 & 11.75 \\
\hline
\end{tabular}

Note-T $=$ trials beyond peak $C R$. 
a significant main effect for pairing $[F(2,78)=3.79]$. Further analyses revealed that the only group to differ from its control was the two-postpeak paired condition $[\mathrm{t}(26)=2.81]$.

Since the two-postpeak unpaired group did not differ significantly from the completely unpaired control, it seems that just two unpaired trials beyond the peak diminishes any conditioning effects during extinction. With respect to the groups that received 16 trials past the peak, pairing or unpairing did not make a difference, since neither of them differed significantly from the unpaired control. As compared with the " 2 " conditions, the substantially smaller number of trials to criterion for the " 16 " conditions was significant in an overall analysis of variance $[F(1,78)=5.80]$.

The extinction data raise the possibility that unpaired trials immediately after the peak substantially accelerate CR attenuation. This can best be examined during acquisition by comparing SCR magnitudes immediately after the peak CR. Since the 2-postpeak conditions allow examination of only 2 such acquisition trials, CR magnitudes for the 16-postpeak conditions were used. These acquisition data, together with those of the completely unpaired group, are presented in Figure 1.

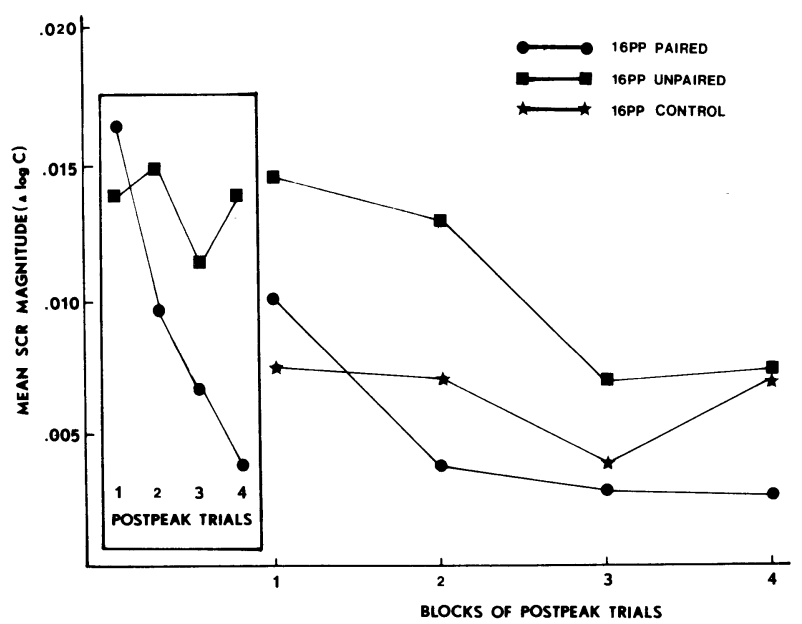

Figure 1. Mean CR magnitudes across four blocks of 16 postpeak acquisition trials for three groups that received 16 paired and unpaired acquisition trials beyond the peak and the matched unpaired control group. Insert shows the mean CR magnitudes across the first four individual postpeak acquisition trials for the two conditioning groups.
Pairing beyond the peak resulted in a slower rate of CR attenuation compared with unpaired trials, which enhanced the attenuation process eventually to a point below the magnitude of the responses of the completely unpaired condition. For most of the trial blocks, the totally paired group demonstrated the largest responses. An analysis of variance revealed that the main effects for groups $[\mathrm{F}(2,39)=3.50, \mathrm{MSe}=.00013]$ and trials $[\mathrm{F}(3,117)=9.70, \mathrm{MSe}=.000023]$ were significant, as was the Groups by Trials interaction $[F(6,117)=3.79$, $\mathrm{MSe}=.000023]$.

A closer examination of first trial block in the above postpeak comparison shows the postpeak unpaired group with lower CR magnitudes than the paired group. However, an examination of the individual four acquisition trials that make up the first trial block reveals the mean magnitude of the first of these four to be approximately the same for both the postpeak paired and unpaired groups (.01465 and .01630 micromhos, respectively). Across the next three individual acquisition trials, the effect of introducing unpaired trials was such that there was a drop of $93 \%$ in the CR magnitude when the first trial beyond the peak was compared with the fourth. Comparing the same two trials, a decrease of only $23 \%$ was observed for the postpeak paired group.

Introducing an extinction procedure just after the peak might produce the same result as the postpeak unpaired group, since in both cases the UCS is no longer paired with the CS. Table 2 shows the mean CR magnitudes for the 2- and 16-postpeak paired groups for four blocks of four trials following the peak. The last 14 trials were extinction trials for the two-postpeak paired condition. Despite the introduction of an extinction procedure, and unlike the group that received postpeak unpaired trials, the 2-postpeak group did not demonstrate smaller mean CR magnitudes than the 16-postpeak condition. Although the mean CR magnitudes for the 2-postpeak group were actually larger on three of the trial blocks, there was no statistical difference between the two groups.

Diminution of unconditioned response occurred across four blocks of four trials each for all three groups in which the UCS continued to be presented, although the groups did not differ in the rate of diminution. An overall trials effect across the four blocks was obtained when 16-postpeak paired, 16-postpeak unpaired, and control groups were compared $[\mathrm{F}(3,117)=14.60$, $\mathrm{MSe}=.000232]$.

Table 2

Mean CR Magnitudes for the 2- and 16-Postpeak Paired Groups Across Four Blocks of Trials

Trial Blocks Beyond Peak

\begin{tabular}{|c|c|c|c|c|c|c|c|c|}
\hline \multirow[b]{2}{*}{ Group } & \multicolumn{2}{|c|}{1} & \multicolumn{2}{|c|}{2} & \multicolumn{2}{|c|}{3} & \multicolumn{2}{|c|}{4} \\
\hline & Mean & SD & Mean & SD & Mean & SD & Mean & SD \\
\hline $\begin{array}{r}\text { 2PP-P } \\
\text { 16PP-P }\end{array}$ & $\begin{array}{l}.0199 \\
.0144\end{array}$ & $\begin{array}{l}.0148 \\
.0328\end{array}$ & $\begin{array}{l}.0107 \\
.0128\end{array}$ & $\begin{array}{l}.0148 \\
.0117\end{array}$ & $\begin{array}{l}.0112 \\
.0065\end{array}$ & $\begin{array}{l}.0174 \\
.0067\end{array}$ & $\begin{array}{l}.0108 \\
.0069\end{array}$ & $\begin{array}{l}.0165 \\
.0051\end{array}$ \\
\hline
\end{tabular}




\section{DISCUSSION}

The postpeak phenomenon is defined as strong resistance to extinction following few acquisition trials. Specifically, 2 paired trials beyond the peak produces significantly more resistance. to extinction than 16 paired trials. The results of the present experiment verified this phenomenon. One possible explanation for this strong resistance to extinction could simply involve extended $C R$ strength during extinction, since the procedure is begun close to peak CR strength. On the other hand, beginning extinction 16 trials beyond the peak involves introducing an extinction procedure at a point at which considerable CR attenuation has already taken place, with the result that the SCR shows relatively weak resistance to extinction. Inherent in this reasoning is the idea that trials beyond the peak involve a relatively constant rate of $C R$ attenuation, independent of the experimental procedure introduced just after the peak. This seems to challenge the traditional view of extinction, which might predict a postpeak acceleration in the rate of CR attenuation. The fact that this does not happen is in itself significant.

However, the results of the present experiment question the notion of a "constant rate" of CR attenuation and, therefore, require an alternative explanation. This becomes necessary since it was demonstrated that during extinction only the twopostpeak paired group shows significant persistence in CR occurrence. When trials beyond the peak are unpaired rather than paired, there is no significant difference between the 2 and 16 groups during extinction. The inability of the postpeak unpaired groups to demonstrate either a 2-16 or conditioning effect during extinction can best be viewed by examining the acquisition data just after the peak. Such an examination reveals that unpaired trials beyond the peak produce a more rapid rate of CR attenuation. On the other hand, introducing extinction just after the peak maintains CR strength in much the same way as paired trials do. It is almost as if the postpeak unpaired condition was more like extinction, while extinction in the two-postpeak paired condition was more like the continuation of conditioning.

The comparison of unpaired and extinction trials beyond the peak involved an extinction procedure begun at two trials past the peak, whereas unpaired trials were begun immediately after the peak. Despite the reduced CR magnitude levels incurred for the extinction group during those two extra postpeak paired trials, relatively larger CR magnitudes were maintained. It is also difficult to argue that maintenance of relatively strong CR magnitudes and strong resistance to extinction in the twopostpeak paired condition is due to the orienting components that result when extinction is introduced just after pairing, since changing from a paired to an unpaired procedure does not have the same result.

Extinction can be viewed as involving both the nonassociation and the removal of the UCS. When extinction is begun just after the peak and strong persistence of the CR results, one of these two variables is responsible. The results of the present study seem to favor the removal of the UCS, since the pairedextinction transfer proved to result in much stronger responding to the CS.

With any extinction procedure in which the UCS is removed and the CS continues to be presented, traces of the UCS occur and, with time, they become weak. However, it has been well established that diminution of the unconditioned response
(Kimmel, 1966) occurs during SCR conditioning, as it did in the present experiment, and that there might be more arousal and discomfort associated with the first few UCS presentations. Evidence for this comes from a recent study in which Silver and Greco (1975) examined the effects of prior direct classical conditioning on a subsequent vicarious classical conditioning situation. Subjects either received 2 or 16 postpeak paired trials and then watched a "model" who supposedly received pairings of the same CS and UCS. Not only did subjects in the " 2 " condition make larger responses to the CS and UCS "received" by the model, but they also judged the same model as experiencing more discomfort than subjects in the " 16 " condition. While continued presentations of the UCS does not seem to systematically affect the felt intensity of the UCS (Kimmel \& Schultz, 1964), it does seem to affect the amount of arousal or anxiety that, with extended UCS presentations, habituates out.

The present experiment calls into question the notion of habituation beyond the peak as being a satisfactory explanation for strong resistance to extinction, since rates of habituation seem to differ beyond the peak depending upon the presence or omission of the UCS. Moreover, the suggestion of a stronger or more arousal-producing UCS as being responsible for strong resistance to extinction following few acquisition trials is supported.

\section{REFERENCES}

KImmeL, H. D. Inhibition of the unconditioned response in classical conditioning. Psychological Review, 1966, 73, 232-240. Kimmel, H. D., \& Schultz, C. A., JR. GSR magnitude and judgements of shock intensity as a function of physical intensity of shock. Psychonomic Science, 1964, 1, 17-18.

Schramm, C. F., \& Kimmel, H. D. Resistance to extinction in GSR conditioning following different numbers of postpeak acquisition trials. Journal of Experimental Psychology, 1970, 84, 239-243.

Silver, A. I. Effects of prior CS presentations on classical conditioning of the skin conductance response. Psychophysiology, 1973, 10, 583-588.

Silver, A. I. Recent developments in classical conditioning of the galvanic skin response: Implications for the acquisition of anxiety. Journal of Behavior Therapy and Experimental Psychiatry, 1977, 8, 337-338.

Silver, A. I., \& Greco, T. A comparison of the effects of vicariously instigated classical conditioning and direct classical conditioning procedures. Pavlovian Journal of Biological Science, 1975, 10, 216-225.

Silver, A. I., \& Kimmel, H. D. Resistance to extinction in classical GSR conditioning as a function of acquisition trials beyond peak CR size. Psychonomic Science, 1969, 14, 53-54.

\section{NOTE}

1. CR attenuation has been used synonymously with habituation. For the purposes of this article, both simply refer to a decrement in responding.

(Received for publication August 17, 1981.) 